\title{
Control of Vibronic Transition Rates by Resonant Single-Molecule-Nanoantenna Coupling
}

\author{
Lisa Saemisch $^{1}$, Matz Liebel ${ }^{1}$ and Niek F. van Hulst ${ }^{1,2^{*}}$ \\ ${ }^{1}$ ICFO-Institut de Ciencies Fotoniques, The Barcelona Institute of Science and Technology, \\ 08860 Castelldefels, Barcelona, Spain \\ ${ }^{2}$ ICREA, Passeig Lluís Companys 23, 08010 Barcelona, Spain
}

\begin{abstract}
KEYWORDS: Spectral Shaping - Vibronic Transition Rate - Antenna Enhancement Emission Spectroscopy - Plasmonics - Localization Microscopy - Nanoantenna Superresolution - Local Density of States
\end{abstract}

\begin{abstract}
Plasmonic nanostructures dramatically alter the radiative and non-radiative properties of single molecules in their vicinity. This coupling induced change in decay channels selectively enhances specific vibronic transitions, which can enable plasmonic control of molecular reactivity. Here, we report coupling dependent spectral emission shaping of single Rhodamine 800 molecules in the vicinity of plasmonic gold nanorods. We show that the relative vibronic transition rates of the first two vibronic transitions of the spontaneous emission spectrum can be tuned in the weak coupling regime, by approximately 25-fold, on the single molecule level.
\end{abstract}


Plasmonic structures manipulate electro-magnetic fields at the nanoscale, by squeezing free space propagating light into nanometric hotspots, and by modifying the local density of states (LDOS) in their vicinity ${ }^{1,2}$. Responsible for these effects are collective oscillations of conduction band electrons, known as localized surface plasmon resonances (LSPR) ${ }^{3}$. Beyond the fundamental importance of light-matter interactions at the nanoscale, a wide range of applications is found in single photon sources ${ }^{4-6}$, optical data storage ${ }^{7}$, photonic circuits ${ }^{8}$, enhanced spectroscopy and sensing $^{9,10}$ as well as solar light detection and catalysis ${ }^{11,12}$.

Lately plasmonic structures are increasingly being used for manipulating and controlling chemical reactions ${ }^{13}$. The combination of nanometric control with the well-known catalytic activity of noble metals allows performing chemical reactions in defined reaction volumes and enables studying chemical reactivity, even on the single-catalyst level ${ }^{14-16}$. Beyond understanding mechanistic details, precisely defined reactant-nanostructure interactions should allow the active control of chemical reactions ${ }^{17}$.

Single emitters in the vicinity of plasmonic nanostructures can be regarded as a test-case for such active interaction control, where the emitters experience a dramatic change in their radiative and non-radiative properties. Strong fluorescence enhancement ${ }^{18,19}$, lifetime shortening ${ }^{18,20}$ and quantum yield changes ${ }^{18,19,21}$ have been observed and these effects will impact the overall emission spectrum through the selective control of individual vibronic spontaneous emission rates ${ }^{22,23}$. Surprisingly, interaction-induced spectral-reshaping has remained largely unexplored and most of our understanding is based on ensemble-based experiments ${ }^{24,25}$ where it is difficult to distinguish overall spectral shifts from the, potentially, dominating contribution of individual, highly-coupled, emitters. 
Ringler and co-workers were the first to report on strong spectral changes of molecules, chemically linked to the hotspot of a nanosphere-dimer ${ }^{24}$. Recently, Lee et al. presented averaged singlemolecule emission spectra near a single spherical gold-nanoparticle ${ }^{26}$. Using localization microscopy, they reported spectral changes for the shorter-distance molecule-particle systems. Yet the spectral effects were subtle, probably due to too large distances and use of a single spherical particle, instead of a gap. In our work, we address the need of a more versatile approach to achieve strong shaping of the spectra of single molecules. First, we use top-down fabricated gold nanorods that we spectrally tune across the molecular resonance (Figure 1a). Second, we operate in liquid, which allows the molecule to probe the hot spot zone, and our wide-field approach allows for the acquisition of a large amount of single-molecule events of different interaction strengths. Selecting the stronger interactions we show that the fluorescence spectrum of single Rhodamine800 (Rho800) molecules can be dramatically altered by the interaction with the top-down fabricated antennas. 
a

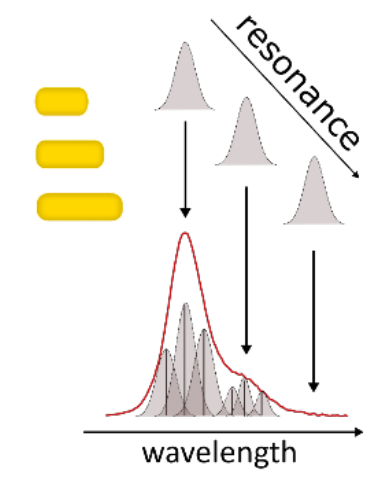

C

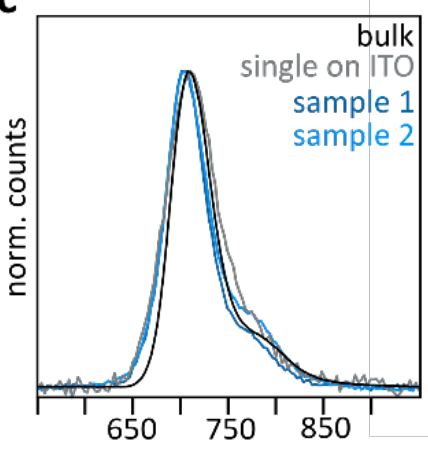

wavelength $(\mathrm{nm})$ b

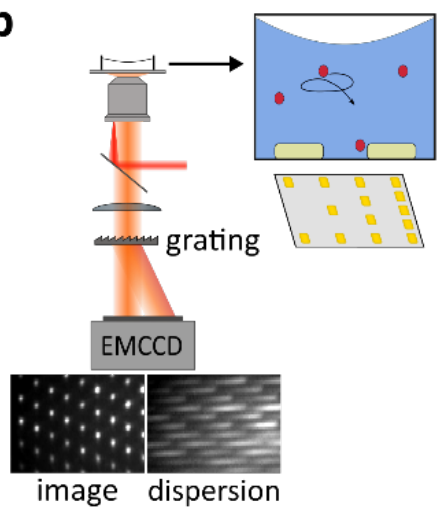

d

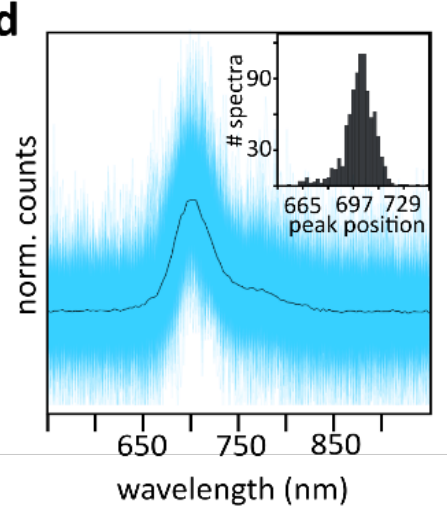

Figure 1, Single-molecule spectral emission control by plasmonic structures. a) The nanorodlength dependent antenna spectrum selectively enhances a subset of vibronic transitions responsible for single-molecule fluorescence emission. b) Schematic of our spectrally-multiplexed widefield microscope alongside a sketch of a typical Au-nanorod sample that is covered with an ethanolic Rho800 solution. c) Comparison of Rho800 spectra measured in bulk solution (black) and as the sum of single Rho800 molecules landing on either bare ITO coverslips (grey) or far away, $>400 \mathrm{~nm}$, from individual Au-antennas on ITO on the nanofabricated samples for two different arrays (light and dark blue); the main 0-0 transition and weak 0-1 side band can be appreciated. d) Plot of all 938 single molecule spectra used for computing the spectral average shown in c) (sample 2) alongside the mean. The inset shows a histogram of the spectral position of the 0-0 transition of all single molecule spectra.

To tune the spectral overlap between molecule and nanoantenna the length of the nanorods is varied from 60 to $150 \mathrm{~nm}$, with a cross-section of 58 x $58 \mathrm{~nm}$ and a separation of $1.5 \mu \mathrm{m}$ between adjacent nanorods. We rely on a home-built total-internal-reflection microscope (Figure 1b) 
equipped with an NA=1.49 objective (Olympus APON60x) and an EM-CCD camera (Andor iXon+ 897e) for wide-field detection, as described previously ${ }^{27}$. The sample is plane-wave illuminated, with $633 \mathrm{~nm}$ linearly polarized light at $5 \mathrm{~kW} / \mathrm{cm}^{2}$ under the critical angle, and the collected single-molecule Rho800 fluorescence is imaged onto the camera. A diffraction grating (Star Analyser SA-100) placed between the camera and the imaging lens enables simultaneous detection of individual emission events alongside their spectra ${ }^{28,29}$. Importantly, the systematic stepwise increase of antenna length on the same sample allows simultaneous imaging of molecular emission spectra in the vicinity of particles with plasmon-resonances ranging from 650 to $850 \mathrm{~nm}$, all through the Rho800 emission band.

Experimentally, we submerge the antenna array in an ethanolic solution of Rho800 and record widefield images at 20 frames per second to capture the stochastically occurring transient emission events of individual molecules ${ }^{27}$. To put our observations into the context of coupling-induced modulations it is imperative to first determine the spectra of uncoupled single molecules and to quantify variations between individual spectra which might be due to a nanoscopically varying dielectric environment ${ }^{30}$. Contrary to previous studies of QDI (quarterrylene diimide) molecules ${ }^{30}$, Rho800 shows only minor deviations between emission from freely diffusing and surface-bound molecules (Figure 1c). Importantly, the fluorescence spectra of 938 individually recorded single molecule events, which were detected spatially separated by $>400 \mathrm{~nm}$ from the nearest gold nanoantenna, are near-identical (Figure 1d).

Following these control-experiments we now focus our attention on emission in the vicinity, $<400$ $\mathrm{nm}$, of the nanoantennas where we initially concentrate on ensemble-spectra obtained by averaging all single molecule events corresponding to a respective antenna-length. We observe an altered behavior with spectral emission changes which are dependent on the precise nanoantenna-length 
(Figure 2). For the shorter antennas, with length between $80-110 \mathrm{~nm}$, the $0-1$ transition of the fluorescence spectrum, around $775 \mathrm{~nm}$, is inhibited with almost all emission occurring via the 0-0 transition. Contrarily, the emission in the vicinity of longer antennas, $120-140 \mathrm{~nm}$, shows enhanced 0-1 emission compared to the uncoupled spectrum (Figure 2). Yet, the changes are small compared to our anticipated calculations.
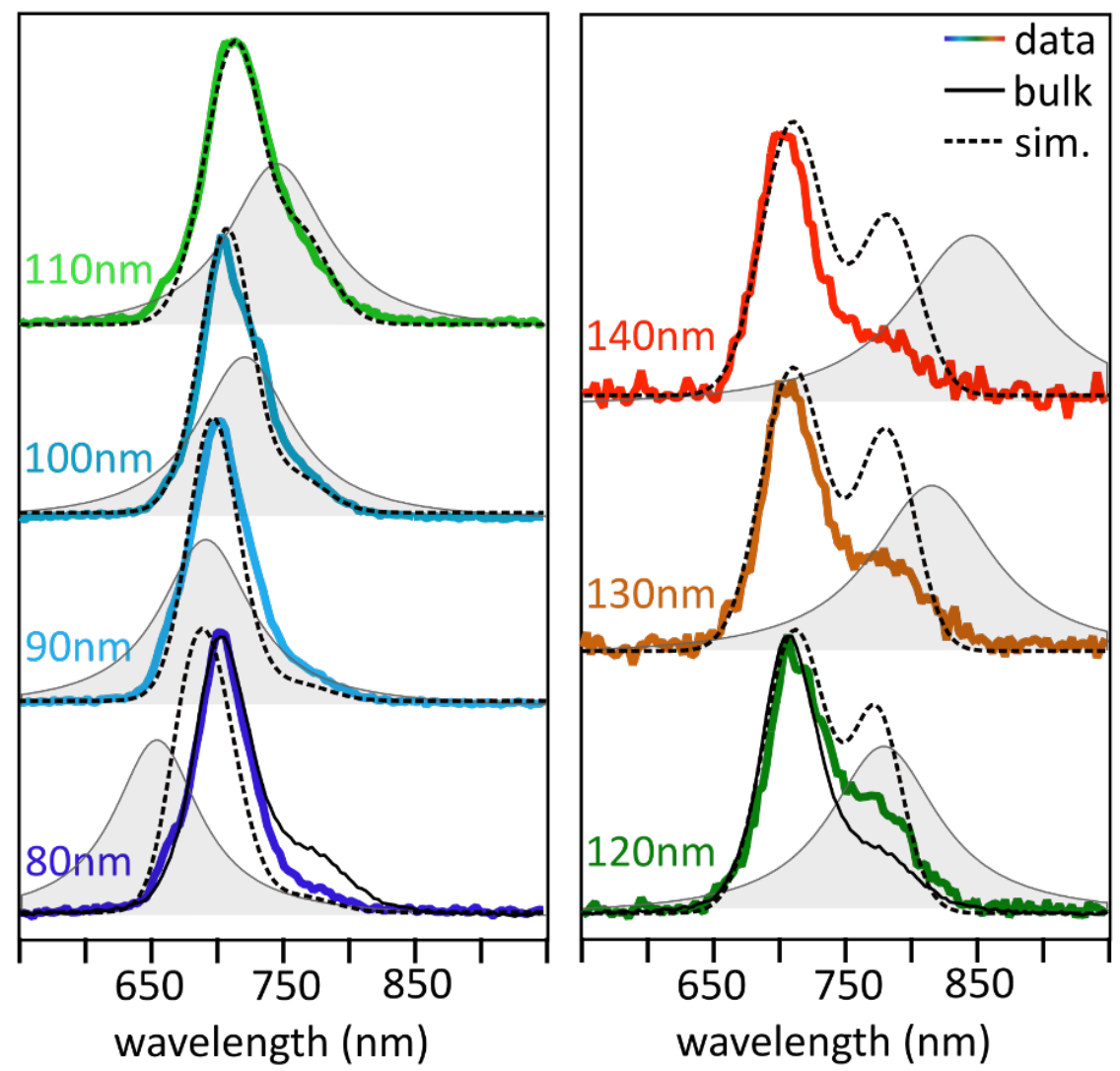

Figure 2, Spectral shaping of molecules next to nanoantennas. Averaged fluorescence spectra of many single molecule events near nanoantennas of different lengths (colored thick lines) alongside normalized extinction spectra of the respective nanoantennas (grey, filled), where we approximate the measured antenna scattering spectra by simple Lorentzians. The unenhanced spectrum of bulk solution (Figure 1c) is indicated with a solid black line. Simulated antennaenhanced emission spectra, obtained by multiplying the unenhanced Rho800 emission with the nanoantenna spectrum, are shown as thin dashed black lines. 
Qualitatively, the emission spectra follow the plasmon-resonance of the gold nanorods, but to further rationalize our observations, we simulate the spectra with a simple coupling-model ${ }^{24}$. Our single-molecule approach allows ignoring fluorescence changes due to excitation enhancement, an important advantage over ensemble-based studies where the total fluorescence is a weighted sum of all contributing molecules and hence a function of the nanoscopically varying field enhancement in the vicinity of the nanostructure. We describe the plasmonically shaped emission spectrum, $F(\omega)$, as a simple sum of all vibronic transition probabilities $t_{n}(\omega)$ multiplied with a nanoantenna-resonance dependent enhancement factor $\sigma_{n}(\omega)$ :

$$
F(\omega)=\sum_{0}^{n} t_{n}(\omega) \cdot \sigma_{n}(\omega)
$$

At room temperature organic molecules exhibit typical homogeneous linewidths of a few hundred wavenumbers, corresponding to electronic dephasing times on the order of a few tenth of femtoseconds ${ }^{31}$ and we, therefore, simplify the coupling model to a multiplication of the antenna spectrum, $S(\omega)$, with the unperturbed molecular emission spectrum, $F_{0}(\omega)$ :

$$
F(\omega)=F_{0}(\omega) \cdot S(\omega)
$$

Here, the nanoantenna scattering spectrum directly relates to its LDOS and thus to the frequency dependent emission enhancement of single emitters in the vicinity of the structure.

The model outlined above qualitatively reproduces the trends in the emission spectra, but suggests 0-0 to $0-1$ ratio changes that are massively off our experimentally obtained results (Figure 2). This difference is due to the fact that we are comparing to ensemble-averaged emission spectra, which naturally include a wide range of antenna-molecule coupling strengths - which reflects directly on the distribution of distances and orientations of emitter and nanoantenna in the ensemble. As a result, weakly coupled molecules, with spectra resembling free Rho800, reduce the shaping effect of the averaged coupling-induced spectra. We record single molecules to avoid this issue, and therefore next we take a closer look at the relevant fraction of stronger coupled molecules. 
a

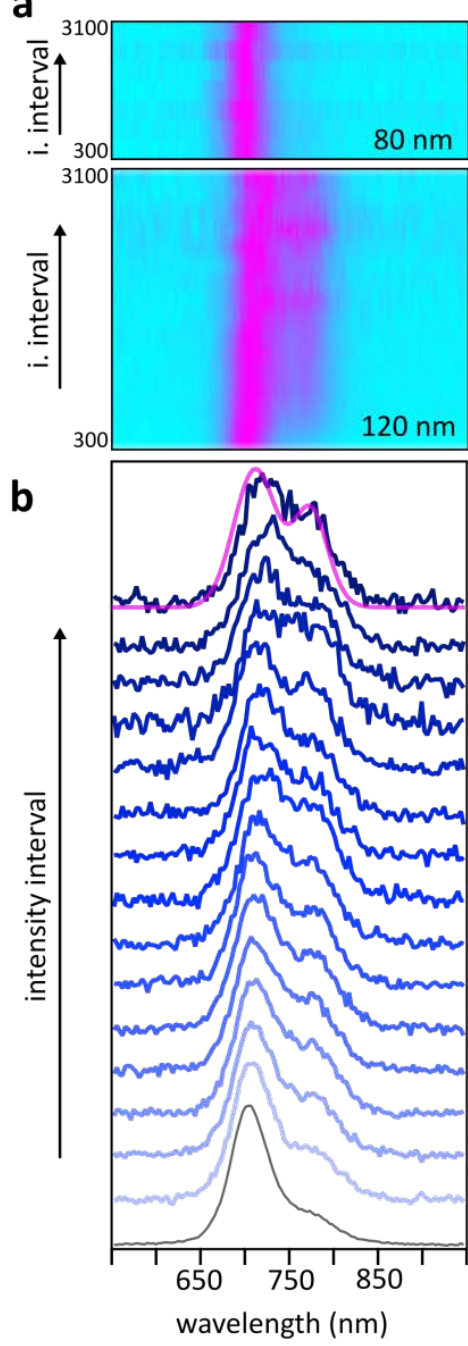

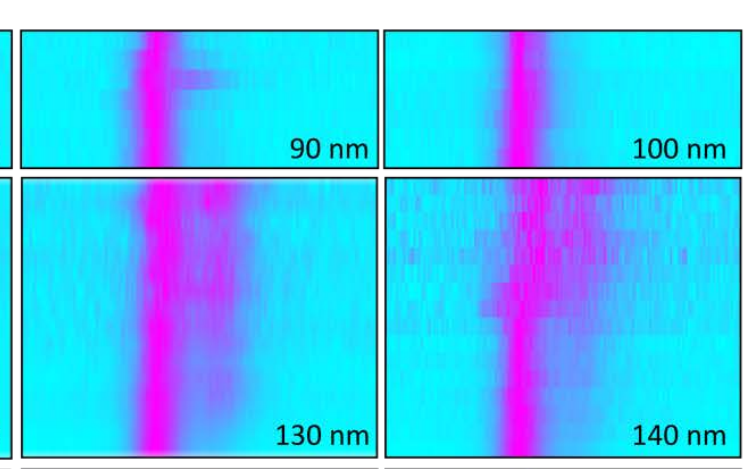
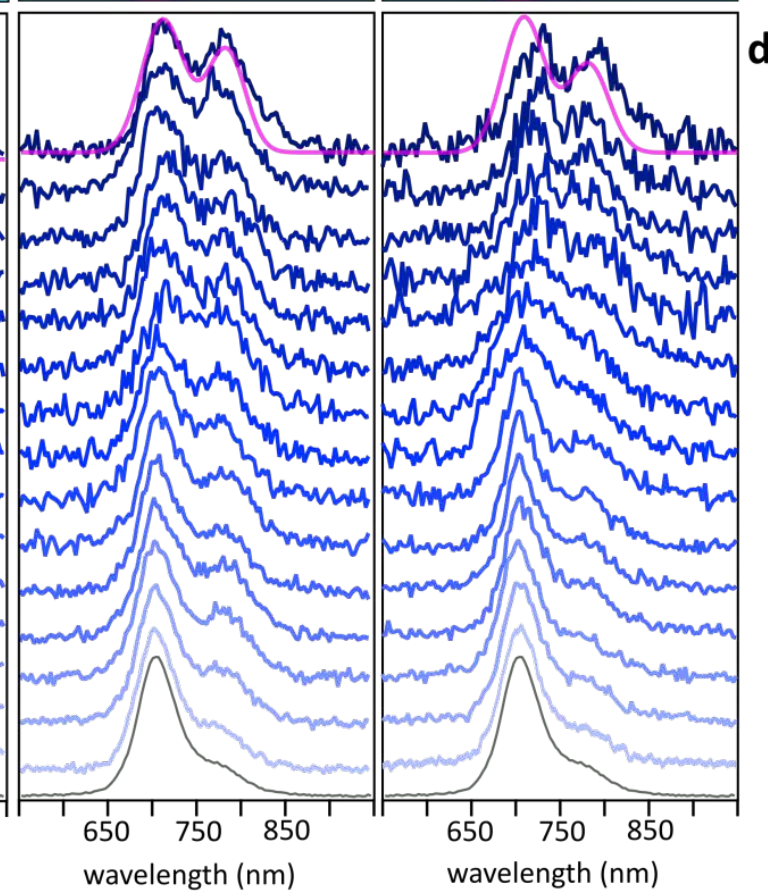

C

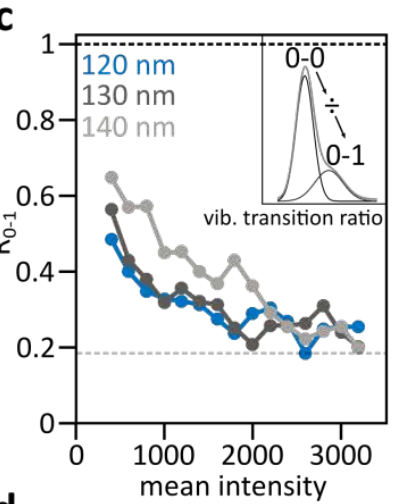

d

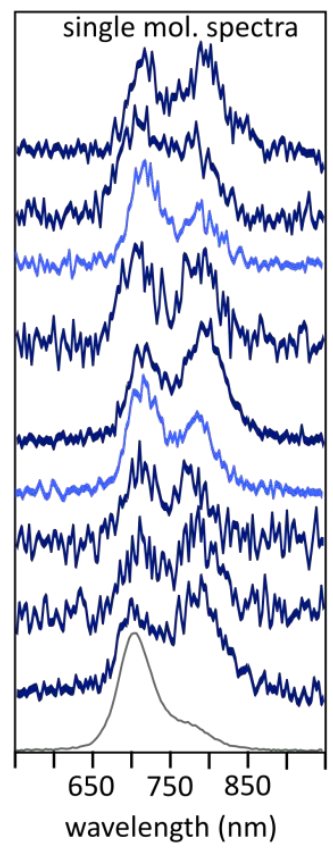

Figure 3, Change of spectra with increasing coupling. a) Spectral evolution with increasing fluorescence emission for nanoantennas of $80-140 \mathrm{~nm}$ in length. For the short nanoantennas $(80-100 \mathrm{~nm})$ the integrated intensity increases in intervals of 400 counts, up to a maximum of 3100 counts; for the longer nanoantennas $(120-140 \mathrm{~nm})$ the intensity increases in intervals of 200 counts, up to a maximum of 3100 counts. The spectra for the shorter antennas are obtained from the previous measurement (Figure 2), and the longer-antenna spectra are measured under the exact same conditions on a second array containing only 120-140 nm antennas. b) Mean spectral evolution (blue) with increasing intensity intervals for the same $120-140 \mathrm{~nm}$ nanoantenna array as in a), compared to the uncoupled spectrum (grey, bottom), and a theoretical prediction of the coupled spectra (pink, top). c) Vibronic transition ratio of the 0-0 to 0-1 spectral bands (see inset), for the spectra presented in b), normalized to the uncoupled Rho800 ratio (black dotted line at ratio 1). The grey dotted line at a value of 0.18 is the ratio below which the 0-1 vibronic sideband transition becomes stronger than the 0-0 transition. d) Selected spectra of interacting single Rho800 molecules next to 120 - $140 \mathrm{~nm}$ nanoantennas. 
We select the spectra of stronger coupled molecules by thresholding the single molecule emission events above a certain intensity level. Even though our measurements are performed in the lowfluence regime the intrinsically low Rho800 fluorescence quantum yield of approximately $0.25^{32}$ allows associating intense emission with antenna-induced radiative rate enhancement and hence stronger coupling ${ }^{27}$. Figure 3a shows the spectral evolution of the $80-100 \mathrm{~nm}$ and $120-140 \mathrm{~nm}$ nanoantennas for intervals of increasing intensity, i.e. stronger coupling. The three short antennas show essentially the 0-0 transition, irrespective of emission intensity, but for longer nanorods we observe a significant spectral evolution when analyzed analogously. We rationalize these trends by considering the spectral overlap between antennas and molecules, as discussed previously. For 80-100 nm antennas, the intrinsically strong 0-0 transition is further enhanced, due to its nearperfect overlap with the antenna-resonance, thus resulting in considerable suppression of the $0-1$ transition, even for relatively weak interactions. For the longer nanorods the opposite trend is observed, favoring the 0-1 interaction. However, the intrinsically low oscillator strength results in only minor changes for weakly coupled emitters. Therefore, only the strongly interacting molecules show considerable spectral deviations from the unenhanced scenario. A direct comparison of the intensity, and hence coupling, dependent spectral evolution with our theoretical prediction shows that it is possible to reach, and even slightly surpass, the predicted degree of spectral shaping (Figure 3b).

To quantify the coupling dependent vibronic decay we define the vibronic transition ratio $R_{0-1}$. To this end we fit the spectra shown in Figure $3 \mathrm{~b}$ to the sum of two Gaussian functions and compute the normalized ratio of the Gaussian amplitudes, equivalent to the vibronic transition ratio

$$
R_{0-1}=\frac{1}{R_{\text {unE }}} * \frac{I_{0-0}}{I_{0-1}}
$$


as a measure of the relative $0-0$ to $0-1$ oscillator strength, with $\mathrm{I}_{0-0}$ and $\mathrm{I}_{0-1}$ being the Gaussian amplitudes and $R_{\text {unE }}=5.5$ a constant that normalizes the ratio to unity for unenhanced molecules. In other words, $\mathrm{R}_{0-1}=1$ corresponds to relative vibronic oscillator strengths reminiscent of an unenhanced molecule, while $\mathrm{R}_{0-1}>1$ implies an enhancement of the main vibronic, the $0-0$, transition and $\mathrm{R}_{0-1}<1$ an enhancement of the $0-1$ transition. The two transitions are of equal strength at a value of $1 / 5.5 \approx 0.18$. To obtain the normalization value of $R_{\text {unE }}=5.5$, we kept the spectral separation between the two Gaussian functions fixed at $70 \mathrm{~nm}$ to ensure a fit to the vibronic sideband and calculated the mean value of the fit to samples 1 and 2 in Figure 1c. We maintained the fixed separation in our analysis, to ensure convergence of the fit also at low signalto-noise ratio. However, owing to the clear presence of two peaks in Figure 3b, we implied only a constraint fit to determine the vibronic transition ratios $R_{0-1}$ in Figure 3c.

The analysis outlined above shows that already the weakest emitters are affected by the presence of the nanorods, showing vibronic transition ratios $R_{0-1}$ reduced from 1.0 to $\sim 0.6$ (Figure 3c). With increasing emission intensity, the ratio changes dramatically, reaching values below 0.2 , which corresponds to the $0-1$ transition being of equal strength as the normally dominating $0-0$ transition, which is a rather interesting scenario where the LDOS of the nanoantenna compensates for the unfavorable wavefunction-projection of the respective vibronic transitions. The ratios discussed thus far are intensity-filtered ensemble spectra but even larger deviations are observed for single molecules (Figure 3d). It should be noted that we simultaneously observe a considerable fraction of strongly emitting molecules with essentially unaltered spectra, thus underlining the need for single-molecule observations to uncover the true spectral-control-potential of the gold nanorod structures employed here. 

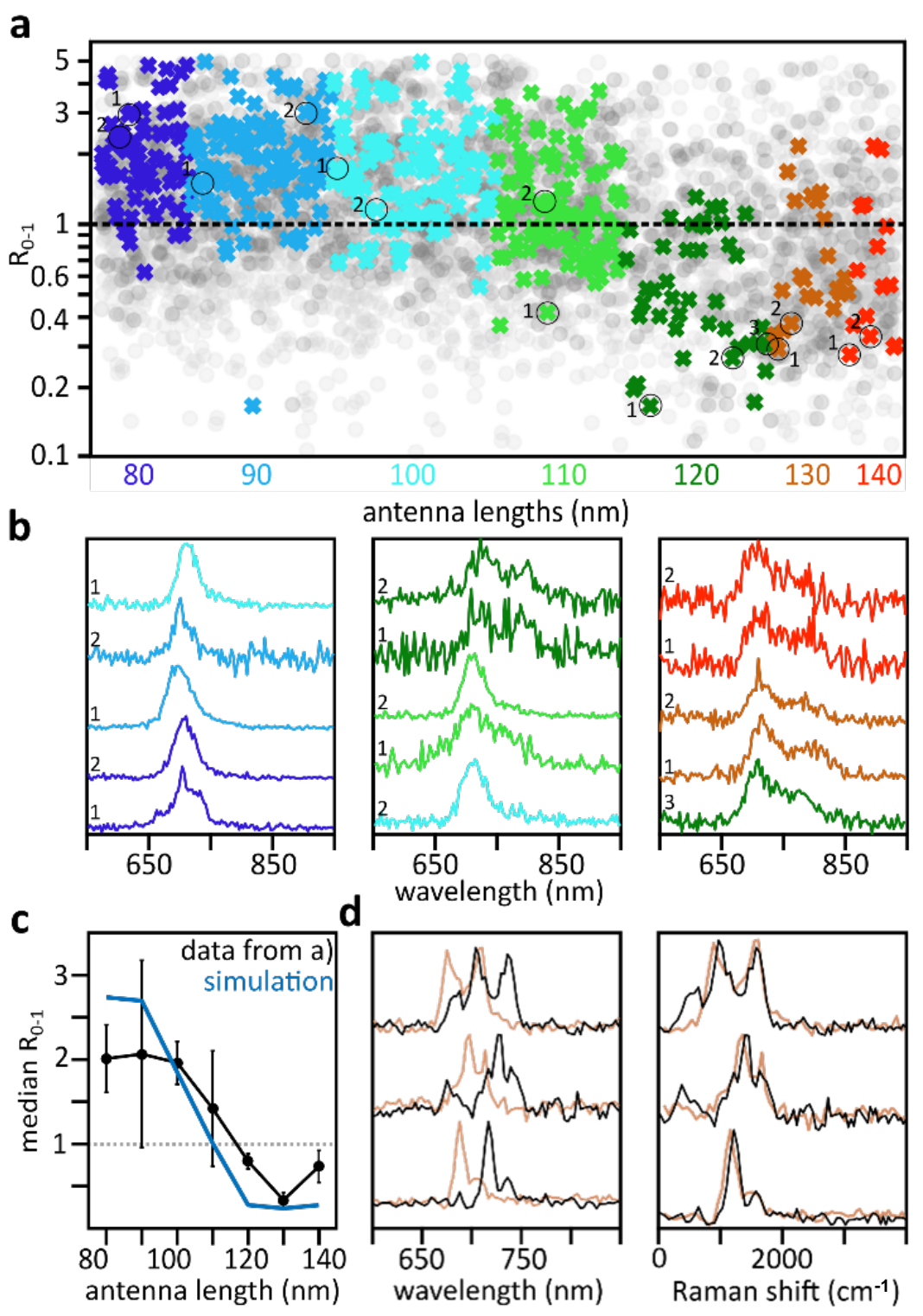

Figure 4, Single molecule vibronic transition ratio and Raman. a) Single molecule 0-0 to 0-1 vibronic transition ratios $R_{0-1}$ sorted according to antenna lengths (all molecules: grey, low intensity threshold: dark grey, high intensity threshold: color-coded). The grey dotted line indicates the normalized ratio of the uncoupled system, $R_{0-1}=1$. B) Selected single molecule spectra, for the molecules indicated by the circles in a). c) Median vibronic transition ratio $R_{0-1}$ for the colored data presented in a), with overlaid simulation-results, for which we approximate the measured antenna scattering spectra by simple Lorentzians to obtain $F(\omega)$ and hence a simulated $\mathrm{R}_{0-1}$. Grey dotted line: uncoupled system. d) Raman spectra: selected Raman spectra of Rho700 molecules for 3 nanoantennas, excited at two different wavelengths of $638 \mathrm{~nm}$ (orange) and $660 \mathrm{~nm}$ (black), and the resulting Raman shift, showing independence on excitation wavelength. 
We conclude with a full single-molecule analysis of the antenna length dependent changes in molecular emission spectra, following the Gaussian fitting-based analysis as outlined above. For the interacting molecules, we determine $R_{0-1}$ (Figure 4a) where a ratio lower (higher) than 1.0 corresponds to 0-1 enhancement (suppression), respectively. As anticipated from the ensemble data (Figure 2) the enhancement gradually moves from being 0-0 to $0-1$ dominant as the nanoantenna-resonances sweep across the Rho800 fluorescence spectrum, with $R_{0-1}$ decreasing from $>5.0$, through 1.0 , to $<0.2$ for certain single molecule encounters. However, a more detailed analysis reveals strong spectral heterogeneity (Figure 4b) albeit good agreement between the population-ensemble and our theoretically expectations (Figure 4c). It should be noted, that the laser excitation wavelength favors the shorter nanorod spectral resonance; therefore, we observe fewer high intensity spectra for the longer nanoantennas. Beyond strongly differing fluorescence spectra obtained for identical nanoantennas we occasionally observe strong spectral modulations, in the form of sharp transitions, reminiscent of strong coupling induced spectral reshaping ${ }^{33}$. These modulations are surprising, as our plasmonic cavities, in the form of simple rod-antennas, should not support the ultra-small mode volume necessary to reach the strong coupling regime. Furthermore, in the advent of strong coupling we would expect fluorescence quenching, while experimentally we observe the opposite. One possible explanation could be single molecule surface enhanced Raman scattering (SERS) due to individual molecules landing at favorable hotspot position on the nanoantenna, i.e. a SERS "hot particle" ${ }^{34}$.

To test this hypothesis, we repeat the experiments described above, yet with two different illumination wavelengths at 638 and $660 \mathrm{~nm}$. We synchronize sample illumination and fluorescence detection to ensure that consecutively record images are illuminated at either $638 \mathrm{~nm}$ or $660 \mathrm{~nm}$, both on the same antenna. Under these experimental conditions signal due to Raman 
scattering will spectrally shift by approximately $520 \mathrm{~cm}^{-1}$ whereas fluorescence emission should be spectrally unaffected. These measurements rely on our capability to record single molecule spectra in two consecutive frames, which is difficult to achieve with Rho800, which shows predominantly transient single molecule emission events. However, Rhodamine 700 (Rho700), which is structurally almost identical to Rho800, seems to exhibit slightly altered surface interactions, which often results in temporally longer lasting emission events. The favorable overlap of laser emissions and Rho700 absorption also contributes to the enhanced probability of encountering SERS signal. Therefore, we perform the two-color experiments with said molecule. Figure 4d shows representative spectra of strongly split, strong-coupling like, molecular emission of Rho700, which seems to be illumination wavelength dependent. After correcting for the illumination wavenumber, we indeed observe near-identical spectra and therefore conclude that the spikes observed in both Figure $4 \mathrm{~b}$ and Figure $4 \mathrm{~d}$ must be attributed to single molecule SERS of favorably bound molecules.

In summary, we measured the fluorescence spectra of single Rho800 molecules in the vicinity of gold nanorods with precisely defined spectral resonances that we tune across the Rho800 emission. The spectrally dependent antenna-molecule coupling impacts the spontaneous emission rates of individual vibronic transitions, in a frequency-dependent manner, allowing to enhance the vibronic sideband to the level of the zero phonon line. Beyond control of vibronic transition rates, we observe the onset of single-molecule SERS, which is especially pronounced for Rho700, most likely due to favorable gold-molecule interactions.

We qualitatively describe the fluorescence reshaping with a simple model that estimates the anticipated emission spectra by computing the overlap of the nanorods' resonances and an unperturbed Rho800 spectrum. The model near-quantitatively reproduces the spectra of strongly 
coupled, intensely emitting, molecules but underestimates the transition perturbations on an ensemble level, which is unsurprising given the expected heterogeneity in interaction strength.

Our single molecule work suggests that it is possible to tune the relative rates of the $0-0$ and $0-1$ transitions by approximately 25 -fold ( $5 \mathrm{x}$ up, $5 \mathrm{x}$ down). Here, either near-perfect suppression of the latter is possible by employing shorter, $80-100 \mathrm{~nm}$, nanorods or, alternatively, we can boost the $0-1$ oscillator strengths up to a point where it acts as the molecules' main decay channel by using longer, 120-140 nm, antennas. We anticipate that, instead of gold, using silver antennas with more narrow resonances, the tuning contrast of 25 -fold can still be substantially enhanced.

Compared to the works of Lee et $a l .{ }^{26}$, we observe a much stronger spectral shaping on a single molecule level, similar to the semi-ensemble work by Ringler et al. ${ }^{24}$. Here, our lithographically fabricated nanoantennas allow to tune to targeted vibronic bands, which we show to be critical within an antenna length difference of 10nm. Moreover, the wide-field detection of an array of antennas provides us with a favorable range of molecule-nanoantenna interactions and ample statistics to analyze the latter; though we cannot precisely determine the exact distance of molecule and nanoantenna.

Our work marks an important step towards nanoscale-manipulation of chemistry at the single molecule level and has direct implications for the rational design of future solar light-harvesting solutions based on plasmonic materials. In this proof-of-concept work we demonstrate discriminating between relative transition-rates with a contrast of approximately $25 x$. We actively control, and shape, different vibronic parts of the same electronic transition but our results should be directly applicable to selectively enhancing one molecular reaction coordinate over another. This control is achieved without requiring strong coupling between the molecule and the metallic 
nanostructure which is an important advantage as it dramatically reduces the hurdles associated with experimentally implementing our control scenario. Furthermore, the weak interaction limit allows rationally designing a suitable plasmonic structure based on the molecules' native reactivity as the wavefunction is only minimally perturbed. Ultimately, we believe that the results presented here pave the way towards LDOS-controlled chemistry and expect successful proof-of-principle demonstrations on a model system such as azobenzene in the near future.

\section{AUTHOR INFORMATION}

\section{Corresponding Author}

* Niek F, van Hulst, e-mail Niek.vanHulst@ICFO.eu. ICFO-Institut de Ciencies Fotoniques, The Barcelona Institute of Science and Technology, 08860 Castelldefels, Barcelona, Spain

\section{Author Contributions}

The experiments were performed by LS and ML; LS analyzed the data and all authors contributed to the manuscript. All authors have given approval to the final version of the manuscript.

\section{Funding Sources}

Authors acknowledge support by the Ministry of Science, Innovation and Universities (MCIU/AEI: BES-2016-078727, RTI2018-099957-J-I00 and PGC2018-096875-B-I00). N.F.v.H. acknowledges the financial support by the European Commission (ERC Advanced Grant 670949LightNet), Ministry of Economy ("Severo Ochoa” program for Centers of Excellence in R\&D SEV-2015-0522 and Plan Nacional FIS2015-69258-P), the Catalan AGAUR (2017SGR1369), Fundació Privada Cellex, Fundació Privada Mir-Puig, and Generalitat de Catalunya through the CERCA program.

\footnotetext{
ABBREVIATIONS

LDOS, local density of states; LSPR, localized surface plasmon resonance; Rho800, Rhodamine 800; Rho700, Rhodamine 700; SERS, surface enhanced Raman scattering; QDI, quarterrylene diimide
} 


\section{REFERENCES}

(1) Novotny, L.; van Hulst, N. F. Antennas for Light. Nat. Photonics 2011, 5 (2), 83-90. https://doi.org/10.1038/nphoton.2010.237.

(2) Muhlschlegel, P.; Eisler, H.-J. H.-J. J.; Martin, O. J. F.; Hecht, B.; Pohl, D. W. Resonant Optical Antennas. Science 2005, 308 (5728), 1607-1609.

https://doi.org/10.1126/science.1111886.

(3) Maier, S. A. Plasmonics - Fundamentals and Applications; Springer Science+Business Media LLC, 2007.

(4) Hoang, T. B.; Akselrod, G. M.; Mikkelsen, M. H. Ultrafast Room-Temperature Single Photon Emission from Quantum Dots Coupled to Plasmonic Nanocavities. Nano Lett. 2016, 16 (1), 270-275. https://doi.org/10.1021/acs.nanolett.5b03724.

(5) Akselrod, G. M.; Argyropoulos, C.; Hoang, T. B.; Ciracì, C.; Fang, C.; Huang, J.; Smith, D. R.; Mikkelsen, M. H. Probing the Mechanisms of Large Purcell Enhancement in Plasmonic Nanoantennas. Nat. Photonics 2014, 8 (11), 835-840. https://doi.org/10.1038/nphoton.2014.228.

(6) Bogdanov, S. I.; Shalaginov, M. Y.; Lagutchev, A. S.; Chiang, C.-C.; Shah, D.; Baburin, A. S.; Ryzhikov, I. A.; Rodionov, I. A.; Kildishev, A. V.; Boltasseva, A.; et al. Ultrabright Room-Temperature Sub-Nanosecond Emission from Single Nitrogen-Vacancy Centers Coupled to Nanopatch Antennas. Nano Lett. 2018, 18 (8), 4837-4844. https://doi.org/10.1021/acs.nanolett.8b01415.

(7) Stipe, B. C.; Strand, T. C.; Poon, C. C.; Balamane, H.; Boone, T. D.; Katine, J. A.; Li, J. L.; Rawat, V.; Nemoto, H.; Hirotsune, A.; et al. Magnetic Recording at 1.5Pbm-2 Using an Integrated Plasmonic Antenna. Nat. Photonics 2010, 4 (7), 484-488. https://doi.org/10.1038/nphoton.2010.90.

(8) Engheta, N. Circuits with Light at Nanoscales: Optical Nanocircuits Inspired by Metamaterials. Science 2007, 317 (5845), 1698-1702. https://doi.org/10.1126/science.1133268.

(9) Willets, K. A.; Van Duyne, R. P. Localized Surface Plasmon Resonance Spectroscopy and Sensing. Annu. Rev. Phys. Chem. 2007, 58 (1), 267-297. https://doi.org/10.1146/annurev.physchem.58.032806.104607.

(10) Mayer, K. M.; Hafner, J. H. Localized Surface Plasmon Resonance Sensors. Chem. Rev. 2011, 111 (6), 3828-3857. https://doi.org/10.1021/cr100313v.

(11) Wu, S.; Sheldon, M. T. Optical Power Conversion via Tunneling of Plasmonic Hot Carriers. ACS Photonics 2018, 5 (6), 2516-2523.

https://doi.org/10.1021/acsphotonics.8b00347. 
(12) Naldoni, A.; Riboni, F.; Guler, U.; Boltasseva, A.; Shalaev, V. M.; Kildishev, A. V. SolarPowered Plasmon-Enhanced Heterogeneous Catalysis. Nanophotonics 2016, 5 (1), 112 133. https://doi.org/10.1515/nanoph-2016-0018.

(13) Baffou, G.; Quidant, R. Nanoplasmonics for Chemistry. Chem. Soc. Rev. 2014, 43 (11), 3898-3907. https://doi.org/10.1039/c3cs60364d.

(14) Zhang, Z.; Zhang, C.; Zheng, H.; Xu, H. Plasmon-Driven Catalysis on Molecules and Nanomaterials. Acc. Chem. Res. 2019, 52 (9), 2506-2515. https://doi.org/10.1021/acs.accounts.9b00224.

(15) Gargiulo, J.; Berté, R.; Li, Y.; Maier, S. A.; Cortés, E. From Optical to Chemical Hot Spots in Plasmonics. Acc. Chem. Res. 2019, 52 (9), 2525-2535. https://doi.org/10.1021/acs.accounts.9b00234.

(16) Simoncelli, S.; Li, Y.; Cortés, E.; Maier, S. A. Nanoscale Control of Molecular SelfAssembly Induced by Plasmonic Hot-Electron Dynamics. ACS Nano 2018, 12 (3), 21842192. https://doi.org/10.1021/acsnano.7b08563.

(17) Galego, J.; Garcia-Vidal, F. J.; Feist, J. Suppressing Photochemical Reactions with Quantized Light Fields. Nat. Commun. 2016, 7, 1-6. https://doi.org/10.1038/ncomms13841.

(18) Wientjes, E.; Renger, J.; Curto, A. G.; Cogdell, R.; van Hulst, N. F. Strong AntennaEnhanced Fluorescence of a Single Light-Harvesting Complex Shows Photon Antibunching. Nat. Commun. 2014, 5, 1-7. https://doi.org/10.1038/ncomms5236.

(19) Kinkhabwala, A.; Yu, Z.; Fan, S.; Avlasevich, Y.; Müllen, K.; Moerner, W. E. Large Single-Molecule Fluorescence Enhancements Produced by a Bowtie Nanoantenna. Nat. Photonics 2009, 3 (11), 654-657. https://doi.org/10.1038/nphoton.2009.187.

(20) Farahani, J. N.; Pohl, D. W.; Eisler, H.-J.; Hecht, B. Single Quantum Dot Coupled to a Scanning Optical Antenna: A Tunable Superemitter. Phys. Rev. Lett. 2005, 95, 017402. https://doi.org/10.1103/PhysRevLett.95.017402.

(21) Yuan, H.; Khatua, S.; Zijlstra, P.; Yorulmaz, M.; Orrit, M. Thousand-Fold Enhancement of Single-Molecule Fluorescence near a Single Gold Nanorod. Angew. Chemie - Int. Ed. 2013, 52 (4), 1217-1221. https://doi.org/10.1002/anie.201208125.

(22) Ming, T.; Chen, H.; Jiang, R.; Li, Q.; Wang, J. Plasmon-Controlled Fluorescence: Beyond the Intensity Enhancement. J. Phys. Chem. Lett. 2012, 3 (2), 191-202. https://doi.org/10.1021/jz201392k.

(23) Curto, A. G.; Volpe, G.; Taminiau, T. H.; Kreuzer, M. P.; Quidant, R.; van Hulst, N. F. Unidirectional Emission of a Quantum Dot Coupled to a Nanoantenna. Science 2010, 329 (5994), 930-933. https://doi.org/10.1126/science.1191922. 
(24) Ringler, M.; Schwemer, A.; Wunderlich, M.; Nichtl, A.; Kürzinger, K.; Klar, T. A.; Feldmann, J. Shaping Emission Spectra of Fluorescent Molecules with Single Plasmonic Nanoresonators. Phys. Rev. Lett. 2008, 100 (20), 1-4. https://doi.org/10.1103/PhysRevLett.100.203002.

(25) Dadosh, T.; Sperling, J.; Bryant, G. W.; Breslow, R.; Shegai, T.; Dyshel, M.; Haran, G.; Bar-Joseph, I. Plasmonic Control of the Shape of the Raman Spectrum of a Single Molecule in a Silver Nanoparticle Dimer. ACS Nano 2009, 3 (7), 1988-1994. https://doi.org/10.1021/nn900422w.

(26) Lee, S. A.; Biteen, J. S. Spectral Reshaping of Single Dye Molecules Coupled to Single Plasmonic Nanoparticles. J.Phys.Chem.Lett. 2019, 10 (19), 5764-5769. https://doi.org/10.1021/acs.jpclett.9b02480.

(27) Saemisch, L.; Liebel, M.; van Hulst, N. F. Statistically Analysis of Many Single-Molecule Encounters Reveals Plasmonic Resonance Dependent Nanoantenna-Molecule Interactions. NanoScale 2020, 12, 3723-3730. https://doi.org/10.1039/c9nr08833d

(28) Zhang, Z.; Kenny, S. J.; Hauser, M.; Li, W.; Xu, K. Ultrahigh-Throughput SingleMolecule Spectroscopy and Spectrally Resolved Super-Resolution Microscopy. Nat. Methods 2015, 12 (10), 935-938. https://doi.org/10.1038/nmeth.3528.

(29) Howell, S. B.; Howell; B., S. Handbook of CCD Astronomy. Handb. CCD Astron. / Steve B. Howell. Cambridge 2000.

(30) Piatkowski, L.; Gellings, E.; van Hulst, N. F. Broadband Single-Molecule Excitation Spectroscopy. Nat. Commun. 2016, 7, 10411. https://doi.org/10.1038/ncomms10411

(31) Troisi, A.; Orlandi, G. Hole Migration in DNA: A Theoretical Analysis of the Role of Structural Fluctuations. J. Phys. Chem. B 2002, 106 (8), 2093-2101. https://doi.org/10.1021/jp0133182.

(32) Alessi, A.; Salvalaggio, M.; Ruzzon, G. Rhodamine 800 as Reference Substance for Fluorescence Quantum Yield Measurements in Deep Red Emission Range. J. Lumin. 2013, 134, 385-389. https://doi.org/10.1016/j.jlumin.2012.08.017.

(33) Hugall, J. T.; Singh, A.; van Hulst, N. F. Plasmonic Cavity Coupling. ACS Photonics 2018, 5 (1), 43-53. https://doi.org/10.1021/acsphotonics.7b01139.

(34) Nie, S.; Emory, S. R. Probing Single Molecules and Single Nanoparticles by Surface Enhanced Raman Scattering. Science 1997, 275, 1102-1106. https://doi.org/10.1126/science.275.5303.1102. 
Table of Contents figure

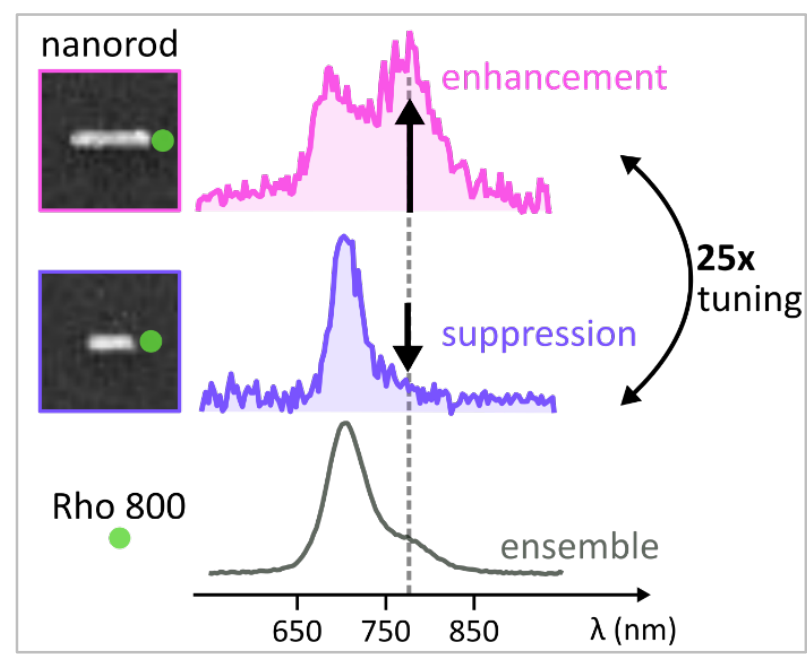

